\title{
Apoptosis, Necrosis, and Necroptosis in the Gut and Intestinal Homeostasis
}

\author{
Anna Negroni, ${ }^{1}$ Salvatore Cucchiara, ${ }^{2}$ and Laura Stronati ${ }^{1}$ \\ ${ }^{1}$ Department of Radiobiology and Human Health, ENEA, Via Anguillarese 301, 00123 Rome, Italy \\ ${ }^{2}$ Department of Pediatrics and Infantile Neuropsychiatry, Pediatric Gastroenterology and Liver Unit, \\ Sapienza University of Rome, Viale Regina Elena 324, 00161 Rome, Italy \\ Correspondence should be addressed to Laura Stronati; laura.stronati@enea.it
}

Received 4 May 2015; Accepted 8 September 2015

Academic Editor: Denis Girard

Copyright (C) 2015 Anna Negroni et al. This is an open access article distributed under the Creative Commons Attribution License, which permits unrestricted use, distribution, and reproduction in any medium, provided the original work is properly cited.

\begin{abstract}
Intestinal epithelial cells (IECs) form a physiochemical barrier that separates the intestinal lumen from the host's internal milieu and is critical for electrolyte passage, nutrient absorption, and interaction with commensal microbiota. Moreover, IECs are strongly involved in the intestinal mucosal inflammatory response as well as in mucosal innate and adaptive immune responses. Cell death in the intestinal barrier is finely controlled, since alterations may lead to severe disorders, including inflammatory diseases. The emerging picture indicates that intestinal epithelial cell death is strictly related to the maintenance of tissue homeostasis. This review is focused on previous reports on different forms of cell death in intestinal epithelium.
\end{abstract}

\section{Introduction}

Intestinal homeostasis depends on complex interactions between microbiota, intestinal epithelium, and host immune system. Diverse regulatory mechanisms cooperate to maintain intestinal homeostasis, and a failure in these pathways may lead to chronic inflammatory disorders [1-3].

The intestinal epithelium represents a huge surface area that is lined by a monolayer of intestinal epithelial cells (IECs), which serve as a barrier to luminal microbes, while also allowing the absorption of water and nutrients essential to life, sensing both beneficial and harmful microbes, and inducing and modulating immune responses [4]. To fulfill such diverse functions, the intestinal epithelium comprises several specialized cell types, divided into two main groups: the absorptive cells, represented by enterocytes, and the secretory cells including Paneth cells, goblet cells, and enteroendocrine cells [5-8]. These subsets of IECs are functionally different and essential to maintain intestinal homeostasis by separating the intestinal lumen from the underlying lamina propria and by controlling the crosstalk between microbiota and subjacent immune cells.
Maintaining barrier function and commensal composition in healthy intestine is also ensured by a basal activation of pattern recognition receptors (PRRs), as Toll-like receptor (TLR) [9], nucleotide oligomerization domain- (NOD-) like receptor (NLR) $[10,11]$, and retinoic acid-inducible gene(RIG-) I-like receptor (RLR) [12] families, able to detect and control various microbial structures. PRRs can activate specific inflammatory transduction signaling that are intimately interconnected with different cell death pathways $[13,14]$, establishing a relationship between host defense mechanisms and cell death.

Intestinal epithelial homeostasis is maintained by a strict equilibrium between cell proliferation in the crypt and cell shedding from the villus tip. In the large and small bowel, differentiated enterocytes are removed constantly and replaced by new cells originated by undifferentiated adult intestinal stem cells, located in the third or fourth position counted from the base of the crypt [15]. These new cells migrate from the base of the crypt to the apical zone of the intestine undergoing maturation. The epithelial layer displays a strict balance between cell proliferation and cell death in order to maintain the intestinal barrier [16]. 

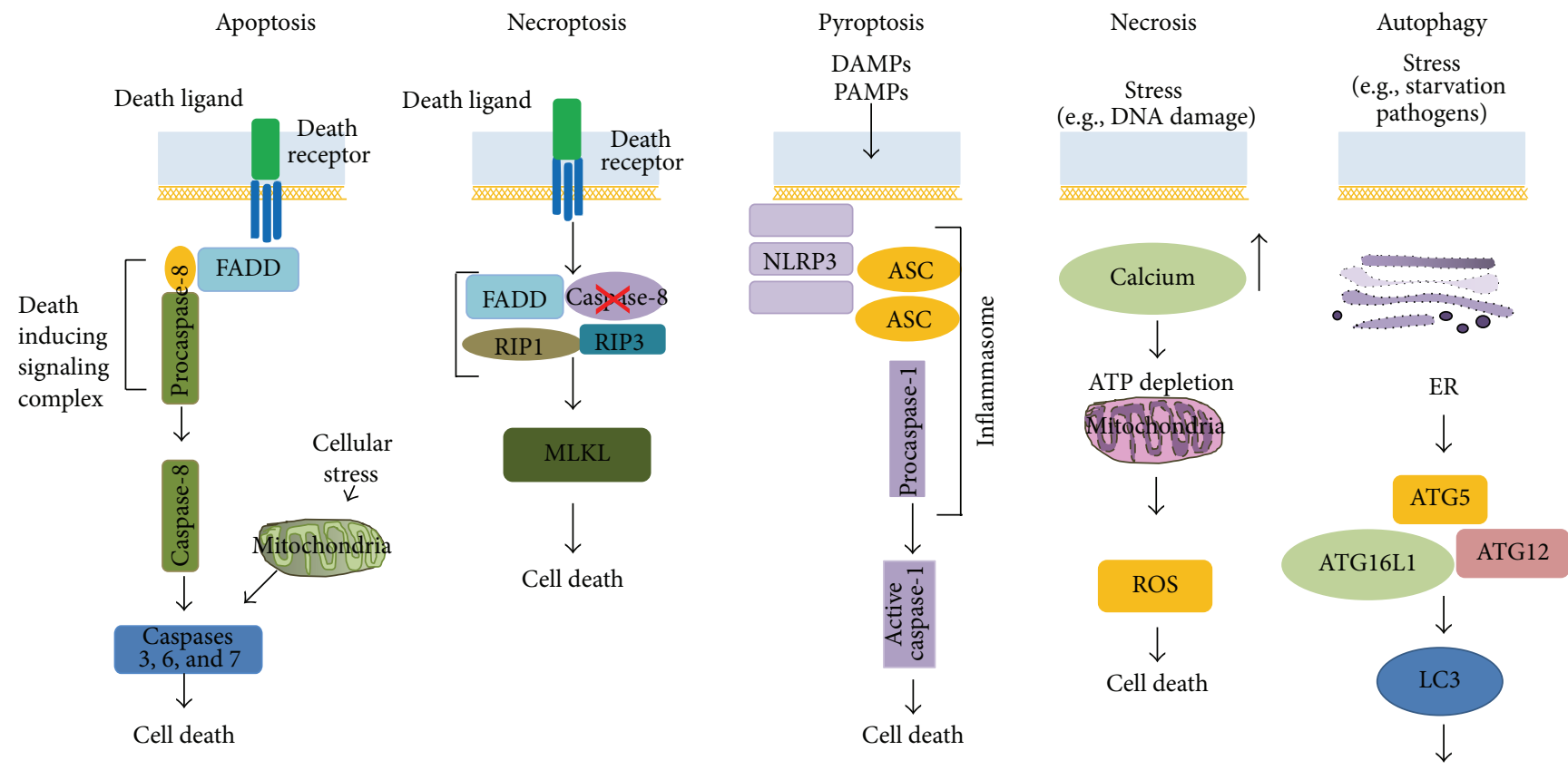

Cell death

FIGURE 1: Cell death pathways. Apoptosis, necroptosis, and pyroptosis are programmed forms of cell death, while necrosis represents an unregulated cell death. Autophagy is a survival pathway that if it is excessive or uncontrolled, it promotes cell death. Fas-associated protein with a death domain (FADD); receptor-interacting interacting protein 1 (RIP1); receptor-interacting interacting protein (RIP3); mixed lineage kinase domain-like (MLKL); danger-associated molecular patterns (DAMPs); pathogen-associated molecular patterns (PAMPs); nod-like receptor family, pyrin domain containing (NLRP)3; apoptosis-associated speck-like protein containing a caspase recruitment domain (ASC); autophagy-related genes (ATG); autophagy related 16-like 1 (ATG16L1); light chain 3 (LC3II); ER: endoplasmic reticulum.

In this review we will describe the relationship between the main forms of epithelial cell death, apoptosis, necrosis and necroptosis, and the intestinal epithelium during gut homeostasis and inflammation. Moreover, two secondary forms of cell death will be shown (Figure 1).

\section{Cell Death and Intestinal Epithelium}

Cell death is a crucial process for tissue development and equilibrium to eliminate superfluous, damaged, or aged cells and represents a key for the homeostasis reestablishment after an acute or chronic insult, limiting the propagation of the inflammatory stimuli to prevent tissue loss of function [17]. This is of particular importance for the gastrointestinal tract, since the intestinal epithelium undergoes continuous and rapid self-renewal, while it is permanently exposed to a plethora of antigens and potential pathogens which are present in the food and in the microbial flora. As a consequence, epithelial cell renewal and cell death need to be tightly regulated because inappropriate cell death responses inexorably lead to the development of diseases, like inflammatory disorders and cancer $[18,19]$. Historically, cell death has been divided in unregulated forms, such as necrosis, and programmed forms, such as apoptosis and necroptosis.

Traditionally, necrosis has been described as a passive and uncontrolled process, initiated by external factors such as ischemia-reperfusion, toxins, viral, and bacterial infections and is characterized by a rapid breakdown of the cell membrane, resulting in the release of intracellular compounds into the extracellular space with activation of the immune system $[20,21]$.

Apoptosis is a process relying on caspase activation that when it is excessive, it may may impair the epithelial barrier, leading to severe gut pathology $[16,22,23]$. Apoptosis has long been considered the only form of regulated cell death, while the existence of additional forms of controlled cell death is now well established [24, 25]. The latter can be triggered independently of apoptosis induction or as back-up safety mechanisms when the apoptotic machinery does not operate properly, as a result of genetic mutations or chemical or microbial inhibition.

Necroptosis is a recently identified form of programmed cell death that is, differently from apoptosis, negatively regulated by caspases and depending on the kinase activity of receptor-interacting proteins (RIP) [26, 27]. Necroptosis shows morphological features similar to necrosis, but, as apoptosis, is strictly regulated by a multiprotein platform. It is characterized by a rapid membrane breakdown, resulting in the release of intracellular compounds, that is, DamageAssociated Molecular Patterns (DAMPs), such as highmobility group box 1 (HMGB1) protein, heat shock proteins, DNA, and RNA, that activate PRRs to further promote an inflammatory response $[25,28]$.

Given the complex structure of the intestinal epithelium, proliferation, differentiation, and cell death must be tightly 
controlled. Excessive cell death might result in a breakdown of the intestinal barrier with subsequent uncontrolled access of bacteria into the gut wall and inflammation [1]. On the contrary, resistance to cell death is believed to be a driving force of tumor development in the gut [28].

\section{Apoptosis}

Apoptosis can be initiated by a wide variety of stimuli including DNA damage, nutrient deficiency, endoplasmic reticulum (ER) stress, growth factor withdrawal, heat shock, developmental cues, and ligation of death-receptors on the cell surface $[22,23]$ through the activation of apoptotic caspases $[23,29]$.

Two sites of epithelial cell death have been described along the length of the villus: the first takes place in the crypt at the level of the stem and early transit cells and is sometimes referred to as "spontaneous" apoptosis and the second at the villus tip or to the surface epithelial cuff in the colon, where epithelial cells, after travelling from the crypt base, differentiate and then die from anoikis [30]. The latter is a special form of programmed cell death induced in anchorage-dependent cells after detachment from their matrix $[31,32]$. Mechanisms control this process are still unsettled [30]. Indeed, cell detachment is suggested to coincide with morphological hallmarks of apoptotic cell death, but a clear causal relationship between cell detachment and apoptosis has not yet been proven [31-33].

A number of studies implicate that cell shedding is actively regulated and involves the proapoptotic molecule caspase-3 $[34,35]$ and that the block of caspases inhibits the tumor necrosis factor- (TNF-) induced cell shedding [35]. Furthermore, it has been reported that TNF or lipopolysaccharide (LPS) stimulation increases apoptosis and consequent cell shedding and is associated with barrier loss [36]. Differently, several studies on mouse models did not support the apoptotic hallmark of shedding epithelial cells. Studies on mice null for proapoptotic molecules, caspase-3, caspase-8, and Fas-associated protein with a death domain (FADD) did not show any morphological alterations in the development of the gastrointestinal epithelium, suggesting that apoptosis is not required for intestinal turnover [37, 38]. Furthermore, experimental data obtained with the use of Necrostatin1, a chemical inhibitor of the kinase receptor interacting protein (RIP)1, showed that murine enterocyte shedding in the small intestine is mostly associated with a nonapoptotic programmed cell death, mediated by RIP1 [39]. Recent studies with RIP1 knock-out mice showed that the loss of caspase8 or TNFR1 completely prevented the intestinal pathology, suggesting a RIP1 essential role in protecting the intestinal epithelia from apoptosis [40-42].

The most convincing hypothesis is that epithelial cell shedding might be a passive process induced by high density of cells in constrained spaces at the villus tip and, thus, the shedding-associated cell death might be a consequence rather than a cause of shedding [43].

Alongside with intestinal cell shedding, patterns of spontaneous apoptosis, p53 mediated, have been described within the crypt region, but with a different regulation between the large and small intestine $[44,45]$. Indeed, it concerns stem cell region in the small intestine but it is rarely found in the colonic crypts. Accordingly, the antiapoptotic gene Bcl-2 (Bcell CLL/lymphoma 2) [45] is barely expressed in the small intestine and strongly expressed at the base of the colonic crypts. Interestingly, differences in $\mathrm{Bcl}-2$ expression and cell death regulation can account for the variability in tumor prevalence between the small and large intestines [46]. Two other studies on mouse models showed that Bcl-2 and Baxnull mice displayed similar levels of spontaneous apoptosis in small intestinal crypts compared to their wild-type strains $[47,48]$.

Altogether these findings highlight that the role of apoptosis in physiology of healthy gut is still controversial.

\section{Apoptosis and Intestinal Inflammation}

An increasing body of evidence suggests that apoptotic signaling may promote inflammatory processes by releasing extracellular vesicles and various chemokines, which may potentially recruit and activate immune cells [49].

Although the role of apoptosis in the structural integrity of the gut is still controversial, there is little doubt that dysregulated or excessive apoptosis can lead to severe gut disorders. Accordingly, several studies reported that mice with elevated apoptosis in the intestinal epithelium more likely develop gut inflammation $[46,50,51]$.

Mice lacking nuclear factor kappa B essential modulator (NEMO) in intestinal epithelial cells (IECs) developed spontaneous colitis shortly after the birth due to excessive TNF-dependent apoptosis, followed by epithelial barrier breakdown and translocation of bacteria into the bowel wall. The inhibition of TNF signaling avoided the development of colitis [52]. Moreover, silencing of other members of the NF$\kappa \mathrm{B}$ pathway, REL-A, transforming growth factor-activated kinase (TAK) 1 or both I $\kappa$ B kinase (IKK) 1 and IKK2, in IECs increased murine susceptibility to spontaneous colitis, providing a strong link among NF- $\kappa \mathrm{B}$ activity, epithelial apoptosis, and intestinal inflammation [53-55].

Furthermore, mice with IEC-specific deletion of the transcription factor Stat3 showed an increased sensitivity to apoptosis upon treatment with dextran sodium sulfate (DSS) [56]. Similarly, XBP1 knock-out mice developed a spontaneous enteritis associated to Paneth cell dysfunction and increased apoptosis of intestinal barrier cells [57].

A dysregulated apoptosis has also been suggested to play a role in the pathogenesis of human inflammatory bowel disease (IBD), including Crohn's disease (CD) and ulcerative colitis (UC). It was reported that an excessive cell shedding and barrier loss in IBD patients in remission predict a disease relapse [58]. Moreover, various apoptotic bodies were found in colonic biopsies routinely taken from patients with active UC, especially in those who required surgery compared with those only treated with medication, suggesting a correlation between IEC apoptosis and disease severity [59]. A twofold increased apoptosis was observed in colonic samples of $\mathrm{CD}$ patients compared to controls. Interestingly, levels of apoptosis returned to control levels when patients underwent 
anti-TNF therapy, suggesting a role of TNF in epithelial cell death $[60,61]$.

T cells in the intestinal mucosa of IBD patients were found to be resistant to multiple apoptotic signals, showing defects in the control of programmed cell death, thus suggesting a possible mechanism to explain why inflammation is resilient to resolution in IBD patients [62-64].

Cell death in the intestinal epithelium seems also to be regulated by bacterial communities. Increased apoptosis has been demonstrated in patients infected with human pathogens, including Salmonella, Shigella, enteropathogenic Escherichia coli, human immunodeficiency virus type 1, Helicobacter pylori, and Cryptosporidium parvum [65-67].

In conclusion, although these studies show a relationship between IBD pathogenesis and altered apoptosis, it is still unclear if the latter is a primary event or a secondary to inflammation.

\section{Necrosis}

Necrosis is considered as an accidental and uncontrolled cell death, frequently associated to disorders such as ischemia-reperfusion (IR) injury, neurodegeneration, intestinal ischemia, and infarction $[20,21,68,69]$. During necrosis, cell and organelles swell and break down with subsequent release of cellular content into the microenvironment, causing the inflammatory response. Common players in necrotic cell death are calcium, which causes mitochondrial calcium overload, bioenergetics effects as well as activation of proteases and phospholipases, and reactive oxygen species (ROS), which produce mitochondrial dysfunction, ion balance deregulation, and loss of membrane integrity [69-71]. Membrane destabilization is also mediated by additional factors, such as acid-sphingomyelinase (ASM), phospholipase A(2) (PLA(2)), and calpains [72]. It has been shown that necrotic cells release immunomodulatory factors causing the activation of the immune response [21, 70, 71].

Necrotic cells have been observed in the colonic epithelium of CD patients, within inflamed as well as uninflamed areas, suggesting that increased necrosis might be a primary, rather than a secondary, mechanism for the development of the disease [73]. Furthermore, the histological analysis revealed, in subjects with IBD, the presence of necrotic intestinal epithelial cells, within the crypts, even during the inactive phase of the disease, implying that necrosis occurs more frequently than expected [74]. In a recent study, the necrosis of Paneth cells in the terminal ileum has been linked to the pathogenesis of IBD [37]. Necrosis as well dysfunction of Paneth cells could explain the decreased production of antimicrobial peptides in IBD [75].

However, whether IEC necrosis normally occurs in the mucosa of the gastrointestinal tract or is strictly associated to inflammation remains still elusive.

Necrosis is also believed to play a fundamental role in infectious gastrointestinal diseases caused by pathogens, since it has been showed that it can be driven by several cytotoxic bacteria [76, 77].

\section{Necroptosis}

Recently, a new caspase-independent mode of programmed cell death, termed necroptosis, has been identified $[31,78,79]$, also in the intestinal epithelium [23,37,38].

Although necroptosis shows morphological features similar to necrosis, it is highly regulated by an intracellular protein platform, largely overlapping with that of apoptosis $[25,26,79-81]$. However, while apoptosis depends on caspase activation, necroptosis is negatively regulated by caspases and needs the intervention of the kinase RIP3 that is thought to be a necroptosis key mediator [82-85]. It is conceivable that when the apoptotic caspases fail to be activated, then cells undergo necroptosis as an alternative death pathway $[79,84]$.

Different endogenous or exogenous stimuli, such as the TNF, ligation of Fas, or the engagement of innate immunity sensors $[79,83]$, may trigger the necroptosis by activating RIP3 [82-85].

Currently, the most informative studies of necroptosis pathway derive from systems that use TNF as a trigger [37, 85, 86], inducing three interrelated signaling pathways, initiated by distinct scaffolding complexes named complex I, complex II, and complex IIb, leading the cell to three different outcomes [23-25]. Upon binding to its receptor, the TNF receptor 1 (TNFR1), the TNF forms the membrane-bound complex I, consisting of the TNF receptor-associated proteins with a death domain (TRADD), the TNFR-associated factor 2 (TRAF2), and RIP1 [86, 87]. After deubiquitination that destabilizes complex I, RIP1 recruits the Fas-associated protein with a death domain (FADD) and caspase- 8 to form complex II, the classical extrinsic apoptotic pathway [87]. If present, RIP3 forms the complex IIb, also known as necrosome [88]. The inhibition of the caspase- 8 and the simultaneous increase of RIP3 promote the activity of the necrosome resulting in necroptosis $[42,84,89]$ (Figure 2).

At present, little is known about the events occurring downstream RIP1 and RIP3 that regulate necroptosis. It is suggested that the RIP homotypic interaction motif (RHIM) on RIP3 and RIP1 allows their interaction and is required for necroptosis induction. Afterwards, RIP1 and RIP3 assemble into cytosolic filamentous structures beta-amyloids, although it is unclear whether they represent a real signaling platform or a postevent accumulation of the two interacting kinases $[21,25,90]$. The RIP1-RIP3 complex drives the RIP3 autophosphorylation which, in turn, phosphorylates the mixed lineage kinase domain-like (MLKL), leading to membrane permeabilization $[88,91-96]$. The occurrence of a crossregulation between apoptotic and necroptotic pathways to maintain cell homeostasis has been suggested; accordingly, necroptosis may act as an emergency back-up death pathway when the apoptotic cascade is impaired [79]. Several in vivo studies demonstrated a role of FADD and caspase- 8 to regulate necroptosis during embryonic development, since excessive necroptosis caused the death of the embryos [97, 98].

Recent papers on knock-out mice showed kinaseindependent RIP1 functions regulating homeostasis and preventing inflammation in barrier tissues by inhibiting epithelial cell apoptosis and necroptosis [40, 41]. Indeed, 


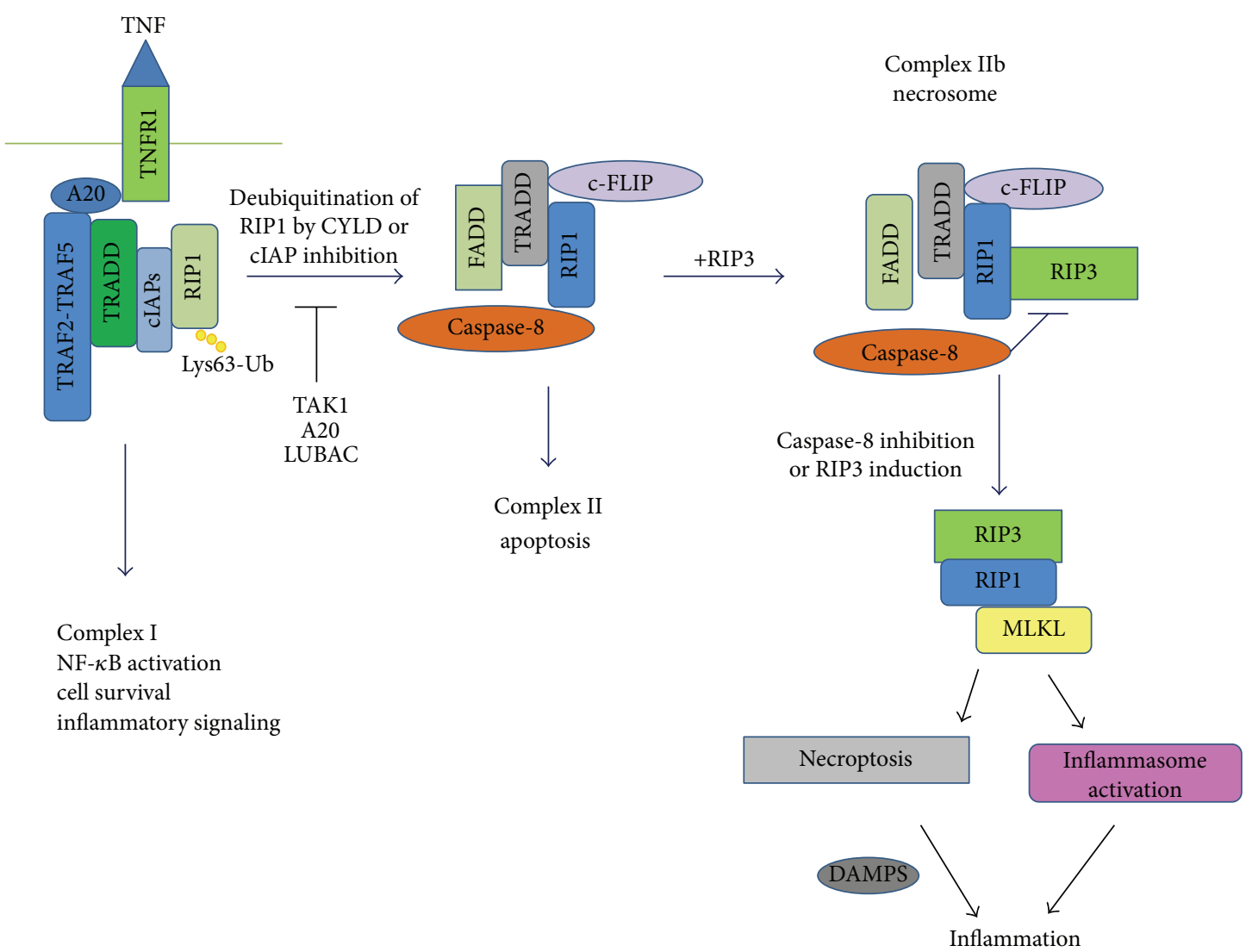

FIGURE 2: TNF-induced programmed cell death pathways. Cell death is induced by various stimuli that are recognized by specific sensors. These receptors recruit the complex I protein platform (TRADD, RIP1, TRAF2, and CIAP1/2), activating the inflammatory response and survival. Deubiquitination of RIP1 induces complex II (FADD and caspase-8), activating the apoptotic pathway. Inhibition of caspase-8 and increase of RIP3 expression induces complex IIb (necrosome), activating the necroptotic pathway. TNF receptor 1 (TNFR1); TNFRassociated death domain (TRADD); receptor-interacting interacting protein 1 (RIP1); receptor-interacting interacting protein (RIP3); TNFRassociated factor 2 (TRAF2); inhibitor of apoptosis proteins 1 and 2 (cIAP1/2); linear ubiquitin chain assembly complex (LUBAC); Lys63-linked polyubiquitination (Lys63-Ub); LUBAC; TAB-transforming growth factor-activated kinase 1 (TAK1); cylindromatosis (CYLD); Fas-associated protein with a death domain (FADD); mixed lineage kinase domain-like (MLKL); Dynamin-related protein (Drp1); dendritic cells (DCs); Damage-Associated Molecular Patterns (DAMPS).

IEC-specific RIP1 deletion caused apoptosis, villus atrophy, loss of goblet and Paneth cells, and premature death in mice. Epithelial FADD ablation inhibited IEC apoptosis and prevented the premature death of mice with IEC-specific RIP1 knockout. However, mice lacking both RIP1 and the apoptotic factor FADD in IECs displayed RIP3-dependent necroptosis, Paneth cell loss, and focal erosive inflammatory lesions in the colon [41].

\section{Necroptosis and Intestinal Inflammation}

Necroptosis, similarly to necrosis, is characterized by the release into the extracellular milieu of immunogenic cytosol content, including alarmins and Damage-Associated Molecular Patterns (DAMPS), that lead to the activation of PRRs, for example, TLRs triggering inflammation [99-102]. Necroptosis has been suggested to play a role in the pathogenesis of several inflammatory disorders, including IBD $[23,27,28,37$, 89].
Caspase- 8 and FADD null mice in IECs exhibited chronic inflammation characterized by extensive epithelial necroptosis with a marked reduction in Paneth cell number and consequent decrease of antimicrobial peptide production [36-38]. However, it is still uncertain whether necroptosis promotes inflammation or if the latter depends on the specific depletion of Paneth cells $[37,75]$. High levels of RIP3 have also been shown in both adult [37] and pediatric [103] CD patients. However, necroptotic triggering factors remain unclear in both mice and humans [101].

\section{Novelties in Cell Death: Pyroptosis and Autophagy}

Pyroptosis, initially described in immune cells during antimicrobial response [104], is a caspase-1 or caspase-11-dependent regulated type of cell death that plays a central role in inflammation and immunity [105-107].

Caspase-1-dependent pyroptosis $[107,108]$, has been better described than caspase-11-dependent pyroptosis [109]. 
Pyroptosis is commenced by the interaction between pathogen-associated molecular patterns (PAMPs) and intracellular PRRs leading to the formation of a multiprotein complex called inflammasome [110] which is composed of dimers of the adaptor protein apoptosis-associated speck-like protein containing a caspase recruitment domain (ASC). A role of RIP3 in activation of inflammasome has also been reported [111]. Four subfamilies of inflammasome have been defined: nucleotide-binding domain, leucine-rich-repeat-containing family, pyrin domain-containing (NLRP)1, NLRP3, NLR family CARD domain-containing protein 4 (NLRC4), and absent in melanoma 2 (AIM2). Currently, the best characterized inflammasome is NLRP3 [112]. Stimulation with specific microbial and endogenous molecules triggers inflammasome assembly and caspase- 1 activation that leads to the secretion of proinflammatory cytokines, including interleukin- $1 \beta$ (IL$1 \beta$ ) or interleukin-18 (IL-18) [110]. In the gut, the inflammasome activation has been largely associated with NODlike receptors and DAMPs that start the enzymatic cascade triggering the inflammatory process [113-115]. The caspase-1 inflammasome is crucial in maintaining intestinal homeostasis. Indeed, mice deficient in NLRP3, NLRP6, NLRC4, ASC, caspase-1, and IL-18 are susceptible to DSS-induced colitis [116]. Interestingly, mice deficient in caspase-11 are also susceptible to DSS-induced colitis, but independently of IL-1 $\beta$ and IL-18 secretion, suggesting the existence of a mechanism distinct from classical inflammasome function in the gut [117, 118]. Indeed, a protective mechanism of caspase-11-dependent pyroptosis in the intestine has been postulated, whose deficiency might drive the disease [119].

Autophagy represents a homeostatic cellular mechanism for the turnover of organelles and proteins, through a lysosome-dependent degradation pathway [120]. During starvation or other stress conditions, autophagy facilitates cell survival through the recycling of metabolic precursors, while excessive or uncontrolled autophagy promotes cell death and morbidity [121]. The concept of autophagic cell death is based on observations of increased morphological features (e.g., accumulation of autophagic vesicles) in dying cells [122]. Targeted cytoplasmic constituents are included in a doublemembraned vesicle known as autophagosome, which is then fused with a lysosome and its cargo degraded and recycled [123]. Some authors suggested that autophagy might play a role in regulating the outcome of other programmed cell death forms as apoptosis, necroptosis, and pyroptosis [114, $121,124]$.

Autophagy-related genes (ATG) are essential regulators of autophagy in development and most other stages of adult life in mice [125]. Homozygous genetic knockouts of most ATG genes (e.g., ATG3, ATG 5, ATG 6, ATG 7, ATG 9, and ATG16L1) in mice are developmentally lethal [125].

Autophagy contributes to the maintenance of intestinal homeostasis, being implicated in nutrient sensing and turnover, as in the control of glucose and amino-acids level and in the recycling of lipids and some micronutrients as iron [126]. A role of autophagy in the modulation of intestinal microbiota and response to bacterial infection has also been recognized [127]. Actually, in intestinal cells, autophagy operates as part of the cell-intrinsic innate immunity program to restrict bacterial replication and dissemination [127].

The identification of the single nucleotide polymorphism (SNP) of the autophagy-related 16-like 1 (ATG16L1) gene, increasing the susceptibility to $\mathrm{CD}$, established a link between autophagy and IBD $[128,129]$. Recently, a defective autophagy has been also related to the activation of inflammasome, induction of pyroptosis, and increased susceptibility to colitis in mouse models $[13,130]$.

\section{Conclusions}

Preserving the integrity of the epithelial barrier by regulating the rate of cell death is considered crucial for maintaining the intestinal homeostasis. Failure of barrier functions, due to an unregulated or excessive cell death, leads to the entry of noxious agents and aberrant stimulation of the intestinal immune system. It is worth noting that the same immune pathways that mediate pathogen recognition and inflammation may themselves trigger cell death, emphasizing the role of the latter in host defense. Besides, the identification of the crossregulatory relationship between different forms of cell death and their intersection with the inflammatory response are fundamental issues to understand their involvement in the development of human intestinal diseases.

\section{Conflict of Interests}

The authors declare that there is no conflict of interests regarding the publication of this paper.

\section{References}

[1] K. J. Maloy and F. Powrie, "Intestinal homeostasis and its breakdown in inflammatory bowel disease," Nature, vol. 474, no. 7351, pp. 298-306, 2011.

[2] W. S. Garrett, J. I. Gordon, and L. H. Glimcher, "Homeostasis and inflammation in the intestine," Cell, vol. 140, no. 6, pp. 859870, 2010.

[3] L. W. Peterson and D. Artis, "Intestinal epithelial cells: regulators of barrier function and immune homeostasis," Nature Reviews Immunology, vol. 14, no. 3, pp. 141-153, 2014.

[4] D. Artis, "Epithelial-cell recognition of commensal bacteria and maintenance of immune homeostasis in the gut," Nature Reviews Immunology, vol. 8, no. 6, pp. 411-420, 2008.

[5] E. Cario, "Toll-like receptors in inflammatory bowel diseases: a decade later," Inflammatory Bowel Diseases, vol. 16, no. 9, pp. 1583-1597, 2010.

[6] W. Strober, P. J. Murray, A. Kitani, and T. Watanabe, "Signalling pathways and molecular interactions of NOD1 and NOD2," Nature Reviews Immunology, vol. 6, no. 1, pp. 9-20, 2006.

[7] R. D. Specian and M. G. Oliver, "Functional biology of intestinal goblet cells," The American Journal of Physiology-Cell Physiology, vol. 260, no. 2, pp. C183-C193, 1991.

[8] T. Sato, J. H. van Es, H. J. Snippert et al., "Paneth cells constitute the niche for Lgr5 stem cells in intestinal crypts," Nature, vol. 469, no. 7330, pp. 415-418, 2011.

[9] M. T. Abreu, "Toll-like receptor signalling in the intestinal epithelium: how bacterial recognition shapes intestinal function," Nature Reviews Immunology, vol. 10, no. 2, pp. 131-144, 2010. 
[10] E. Elinav, J. Henao-Mejia, and R. A. Flavell, "Integrative inflammasome activity in the regulation of intestinal mucosal immune responses," Mucosal Immunology, vol. 6, no. 1, pp. 4-13, 2013.

[11] G. Y. Chen and G. Nez, "Inflammasomes in intestinal inflammation and cancer," Gastroenterology, vol. 141, no. 6, pp. 1986-1999, 2011.

[12] A. H. Broquet, Y. Hirata, C. S. McAllister, and M. F. Kagnoff, "RIG-I/MDA5/MAVS are required to signal a protective IFN response in rotavirus-infected intestinal epithelium," The Journal of Immunology, vol. 186, no. 3, pp. 1618-1626, 2011.

[13] J. M. Blander, "A long-awaited merger of the pathways mediating host defence and programmed cell death," Nature Reviews Immunology, vol. 14, no. 9, pp. 601-618, 2014.

[14] T. T. MacDonald, I. Monteleone, M. C. Fantini, and G. Monteleone, "Regulation of homeostasis and inflammation in the intestine," Gastroenterology, vol. 140, no. 6, pp. 1768-1775, 2011.

[15] C. S. Potten, R. Gandara, Y. R. Mahida, M. Loeffler, and N. A. Wright, "The stem cells of small intestinal crypts: where are they?” Cell Proliferation, vol. 42, no. 6, pp. 731-750, 2009.

[16] K. L. Edelblum, F. Yan, T. Yamaoka, and D. B. Polk, "Regulation of apoptosis during homeostasis and disease in the intestinal epithelium," Inflammatory Bowel Diseases, vol. 12, no. 5, pp. 413424, 2006.

[17] A. Strasser and M. Pellegrini, "T-lymphocyte death during shutdown of an immune response," Trends in Immunology, vol. 25, no. 11, pp. 610-615, 2004.

[18] L. G. Van der Flier and H. Clevers, "Stem cells, self-renewal, and differentiation in the intestinal epithelium," Annual Review of Physiology, vol. 71, pp. 241-260, 2009.

[19] C. Crosnier, D. Stamataki, and J. Lewis, "Organizing cell renewal in the intestine: stem cells, signals and combinatorial control," Nature Reviews Genetics, vol. 7, no. 5, pp. 349-359, 2006.

[20] S. Y. Proskuryakov, A. G. Konoplyannikov, and V. L. Gabai, "Necrosis: a specific form of programmed cell death?" Experimental Cell Research, vol. 283, no. 1, pp. 1-16, 2003.

[21] N. Vanlangenakker, T. Vanden Berghe, D. V. Krysko, N. Festjens, and P. Vandenabeele, "Molecular mechanisms and pathophysiology of necrotic cell death," Current Molecular Medicine, vol. 8, no. 3, pp. 207-220, 2008.

[22] R. C. Taylor, S. P. Cullen, and S. J. Martin, "Apoptosis: controlled demolition at the cellular level," Nature Reviews Molecular Cell Biology, vol. 9, no. 3, pp. 231-241, 2008.

[23] C. Günther, H. Neumann, M. F. Neurath, and C. Becker, "Apoptosis, necrosis and necroptosis: cell death regulation in the intestinal epithelium," Gut, vol. 62, no. 7, pp. 1062-1071, 2013.

[24] S. W. G. Tait, G. Ichim, and D. R. Green, "Die another waynon-apoptotic mechanisms of cell death," Journal of Cell Science, vol. 127, no. 10, pp. 2135-2144, 2014.

[25] T. V. Berghe, A. Linkermann, S. Jouan-Lanhouet, H. Walczak, and P. Vandenabeele, "Regulated necrosis: the expanding network of non-apoptotic cell death pathways," Nature Reviews Molecular Cell Biology, vol. 15, no. 2, pp. 135-147, 2014.

[26] P. Vandenabeele, L. Galluzzi, T. V. Berghe, and G. Kroemer, "Molecular mechanisms of necroptosis: an ordered cellular explosion," Nature Reviews Molecular Cell Biology, vol. 11, no. 10, pp. 700-714, 2010.

[27] M. Pasparakis, "Necroptosis and its role in inflammation," Nature, vol. 517, no. 7534, pp. 311-320, 2015.

[28] M. Dagenais, T. Douglas, and M. Saleh, "Role of programmed necrosis and cell death in intestinal inflammation," Current Opinion in Gastroenterology, vol. 30, no. 6, pp. 566-575, 2014.
[29] C. Becker, A. J. Watson, and M. F. Neurath, "Complex roles of caspases in the pathogenesis of inflammatory bowel disease," Gastroenterology, vol. 144, no. 2, pp. 283-293, 2013.

[30] K. Bertrand, "Survival of exfoliated epithelial cells: a delicate balance between anoikis and apoptosis," Journal of Biomedicine and Biotechnology, vol. 2011, Article ID 534139, 9 pages, 2011.

[31] J. Yuan and G. Kroemer, "Alternative cell death mechanisms in development and beyond," Genes and Development, vol. 24, no. 23, pp. 2592-2602, 2010.

[32] A. J. M. Watson, C. A. Duckworth, Y. Guan, and M. H. Montrose, "Mechanisms of epithelial cell shedding in the mammalian intestine and maintenance of barrier function," Annals of the New York Academy of Sciences, vol. 1165, pp. 135-142, 2009.

[33] P. A. Hall, P. J. Coates, B. Ansari, and D. Hopwood, "Regulation of cell number in the mammalian gastrointestinal tract: the importance of apoptosis," Journal of Cell Science, vol. 107, no. 12, pp. 3569-3577, 1994.

[34] T. F. Bullen, S. Forrest, F. Campbell et al., "Characterization of epithelial cell shedding from human small intestine," Laboratory Investigation, vol. 86, no. 10, pp. 1052-1063, 2006.

[35] A. M. Marchiando, L. Shen, W. V. Graham et al., "The epithelial barrier is maintained by in vivo tight junction expansion during pathologic intestinal epithelial shedding," Gastroenterology, vol. 140, no. 4, pp. 1208-1218, 2011.

[36] C. Günther, B. Buchen, G. He et al., "Caspase- 8 controls the gut response to microbial challenges by Tnf- $\alpha$-dependent and independent pathways," Gut, vol. 64, no. 4, pp. 601-610, 2015.

[37] C. Günther, E. Martini, N. Wittkopf et al., "Caspase-8 regulates TNF-alpha-induced epithelial necroptosis and terminal ileitis," Nature, vol. 477, no. 7364, pp. 335-339, 2011.

[38] P.-S. Welz, A. Wullaert, K. Vlantis et al., "FADD prevents RIP3mediated epithelial cell necrosis and chronic intestinal inflammation," Nature, vol. 477, no. 7364, pp. 330-334, 2011.

[39] Y. Matsuoka and Y. Tsujimoto, "Role of RIP1 in physiological enterocyte turnover in mouse small intestine via nonapoptotic death," Genes to Cells, vol. 20, no. 1, pp. 11-28, 2015.

[40] J. A. Rickard, J. A. O’Donnell, J. M. Evans et al., "RIPK1 regulates RIPK3-MLKL-driven systemic inflammation and emergency hematopoiesis," Cell, vol. 157, no. 5, pp. 1175-1188, 2014.

[41] M. Dannappel, K. Vlantis, S. Kumari et al., "RIPK1 maintains epithelial homeostasis by inhibiting apoptosis and necroptosis," Nature, vol. 513, no. 7516, pp. 90-94, 2014.

[42] K. Newton, D. L. Dugger, K. E. Wickliffe et al., "Activity of protein kinase RIPK3 determines whether cells die by necroptosis or apoptosis," Science, vol. 343, no. 6177, pp. 1357-1360, 2014.

[43] G. T. Eisenhoffer, P. D. Loftus, M. Yoshigi et al., "Crowding induces live cell extrusion to maintain homeostatic cell numbers in epithelia," Nature, vol. 484, no. 7395, pp. 546-549, 2012.

[44] K. H. Vousden and X. Lu, "Live or let die: the cell's response to p53," Nature Reviews Cancer, vol. 2, no. 8, pp. 594-604, 2002.

[45] A. J. Merritt, C. S. Potten, A. J. M. Watson et al., "Differential expression of bcl-2 in intestinal epithelia. Correlation with attenuation of apoptosis in colonic crypts and the incidence of colonic neoplasia," Journal of Cell Science, vol. 108, no. 6, pp. 2261-2271, 1995.

[46] A. G. Renehan, S. P. Bach, and C. S. Potten, "The relevance of apoptosis for cellular homeostasis and tumorigenesis in the intestine," Canadian Journal of Gastroenterology, vol. 15, no. 3, pp. 166-176, 2001.

[47] K. Nakayama, I. Negishi, K. Kuida, K.-I. Nakayama, H. Sawa, and D. Y. Loh, "Targeted disruption of Bcl-2alpha beta in 
mice: occurrence of gray hair, polycystic kidney disease, and lymphocytopenia," Proceedings of the National Academy of Sciences of the United States of America, vol. 91, no. 9, pp. 37003704, 1994.

[48] C. M. Knudson, K. S. K. Tung, W. G. Tourtellotte, G. A. J. Brown, and S. J. Korsmeyer, "Bax-deficient mice with lymphoid hyperplasia and male germ cell death," Science, vol. 270, no. 5233, pp. 96-99, 1995.

[49] A. Canbay, A. E. Feldstein, H. Higuchi et al., "Kupffer cell engulfment of apoptotic bodies stimulates death ligand and cytokine expression," Hepatology, vol. 38, no. 5, pp. 1188-1198, 2003.

[50] S. El Andaloussi, I. Mäger, X. O. Breakefield, and M. J. A. Wood, "Extracellular vesicles: biology and emerging therapeutic opportunities," Nature Reviews Drug Discovery, vol. 12, no. 5, pp. 347-357, 2013.

[51] Y. Fuchs and H. Steller, "Programmed cell death in animal development and disease," Cell, vol. 147, no. 4, pp. 742-758, 2011.

[52] A. Nenci, C. Becker, A. Wullaert et al., "Epithelial NEMO links innate immunity to chronic intestinal inflammation," Nature, vol. 446, no. 7135, pp. 557-561, 2007.

[53] M. Pasparakis, "Regulation of tissue homeostasis by NF-kappaB signalling: implications for inflammatory diseases," Nature Reviews Immunology, vol. 9, no. 11, pp. 778-788, 2009.

[54] R. Kajino-Sakamoto, M. Inagaki, E. Lippert et al., "Enterocytederived TAK1 signaling prevents epithelium apoptosis and the development of ileitis and colitis," Journal of Immunology, vol. 181, no. 2, pp. 1143-1152, 2008.

[55] K. A. Steinbrecher, E. Harmel-Laws, R. Sitcheran, and A. S. Baldwin, "Loss of epithelial RelA results in deregulated intestinal proliferative/apoptotic homeostasis and susceptibility to inflammation," The Journal of Immunology, vol. 180, no. 4, pp. 2588-2599, 2008.

[56] J. Bollrath, T. J. Phesse, V. A. von Burstin et al., "gp130-mediated Stat3 activation in enterocytes regulates cell survival and cell-cycle progression during colitis-associated tumorigenesis," Cancer Cell, vol. 15, no. 2, pp. 91-102, 2009.

[57] A. Kaser, A.-H. Lee, A. Franke et al., "XBP1 links ER stress to intestinal inflammation and confers genetic risk for human inflammatory bowel disease," Cell, vol. 134, no. 5, pp. 743-756, 2008.

[58] R. Kiesslich, C. A. Duckworth, D. Moussata et al., "Local barrier dysfunction identified by confocal laser endomicroscopy predicts relapse in inflammatory bowel disease," Gut, vol. 61, no. 8, pp. 1146-1153, 2012.

[59] C. Hagiwara, M. Tanaka, and H. Kudo, "Increase in colorectal epithelial apoptotic cells in patients with ulcerative colitis ultimately requiring surgery," Journal of Gastroenterology and Hepatology, vol. 17, no. 7, pp. 758-764, 2002.

[60] S. Zeissig, C. Bojarski, N. Buergel et al., "Downregulation of epithelial apoptosis and barrier repair in active Crohn's disease by tumour necrosis factor $\alpha$ antibody treatment," Gut, vol. 53, no. 9, pp. 1295-1302, 2004.

[61] A. Di Sabatino, R. Ciccocioppo, O. Luinetti et al., "Increased enterocyte apoptosis in inflamed areas of Crohn's disease," Diseases of the Colon and Rectum, vol. 46, no. 11, pp. 1498-1507, 2003.

[62] M. Boirivant, M. Marini, G. Di Felice et al., "Lamina propria T cells in Crohn's disease and other gastrointestinal inflammation show defective CD2 pathway-induced apoptosis," Gastroenterology, vol. 116, no. 3, pp. 557-565, 1999.
[63] K. Ina, J. Itoh, K. Fukushima et al., "Resistance of Crohn's disease $\mathrm{T}$ cells to multiple apoptotic signals is associated with a Bcl2/Bax mucosal imbalance," The Journal of Immunology, vol. 163, no. 2, pp. 1081-1090, 1999.

[64] I. Monteleone, G. Monteleone, D. Fina et al., "A functional role of flip in conferring resistance of Crohn's disease lamina propria lymphocytes to FAS-mediated apoptosis," Gastroenterology, vol. 130, no. 2, pp. 389-397, 2006.

[65] A. Ramachandran, M. Madesh, and K. A. Balasubramanian, "Apoptosis in the intestinal epithelium: its relevance in normal and pathophysiological conditions," Journal of Gastroenterology and Hepatology, vol. 15, no. 2, pp. 109-120, 2000.

[66] S. E. Winter and A. J. Bäumler, "Salmonella exploits suicidal behavior of epithelial cells," Frontiers in Microbiology, vol. 2, article 48, 2011.

[67] L. A. Knodler, B. A. Vallance, J. Celli et al., "Dissemination of invasive Salmonella via bacterial-induced extrusion of mucosal epithelia," Proceedings of the National Academy of Sciences of the United States of America, vol. 107, no. 41, pp. 17733-17738, 2010.

[68] N. K. Chokshi, Y. S. Guner, C. J. Hunter, J. S. Upperman, A. Grishin, and H. R. Ford, "The role of nitric oxide in intestinal epithelial injury and restitution in neonatal necrotizing enterocolitis," Seminars in Perinatology, vol. 32, no. 2, pp. 92-99, 2008.

[69] N. Vanlangenakker, T. Vanden Berghe, and P. Vandenabeele, "Many stimuli pull the necrotic trigger: an overview," Cell Death and Differentiation, vol. 19, no. 1, pp. 75-86, 2012.

[70] P. Golstein and G. Kroemer, "Cell death by necrosis: towards a molecular definition," Trends in Biochemical Sciences, vol. 32, no. 1, pp. 37-43, 2007.

[71] N. Festjens, T. Vanden Berghe, and P. Vandenabeele, "Necrosis, a well-orchestrated form of cell demise: signalling cascades, important mediators and concomitant immune response," Biochimica et Biophysica Acta, vol. 1757, no. 9-10, pp. 1371-1387, 2006.

[72] S. Y. Proskuryakov and V. L. Gabai, "Mechanisms of tumor cell necrosis," Current Pharmaceutical Design, vol. 16, no. 1, pp. 5668, 2010.

[73] R. R. Dourmashkin, H. Davies, C. Wells et al., "Epithelial patchy necrosis in Crohn's disease," Human Pathology, vol. 14, no. 7, pp. 643-648, 1983.

[74] D. H. Barkla and P. R. Gibson, "The fate of epithelial cells in the human large intestine," Pathology, vol. 31, no. 3, pp. 230-238, 1999.

[75] K. Lewin, “The Paneth cell in disease," Gut, vol. 10, no. 10, pp. 804-811, 1969.

[76] M. François, V. Le Cabec, M.-A. Dupont, P. J. Sansonetti, and I. Maridonneau-Parini, "Induction of necrosis in human neutrophils by Shigella flexneri requires type III secretion, IpaB and IpaC invasins, and actin polymerization," Infection and Immunity, vol. 68, no. 3, pp. 1289-1296, 2000.

[77] H.-G. Jeong and K. J. F. Satchell, "Additive function of Vibrio vulnificus $\mathrm{MARTX}_{\mathrm{Vv}}$ and $\mathrm{VvhA}$ cytolysins promotes rapid growth and epithelial tissue necrosis during intestinal infection," PLoS Pathogens, vol. 8, no. 3, Article ID e1002581, 13 pages, 2012.

[78] D. E. Christofferson and J. Yuan, "Necroptosis as an alternative form of programmed cell death," Current Opinion in Cell Biology, vol. 22, no. 2, pp. 263-268, 2010.

[79] J. Han, C.-Q. Zhong, and D.-W. Zhang, "Programmed necrosis: backup to and competitor with apoptosis in the immune system," Nature Immunology, vol. 12, no. 12, pp. 1143-1149, 2011. 
[80] T. V. Berghe, N. Vanlangenakker, E. Parthoens et al., "Necroptosis, necrosis and secondary necrosis converge on similar cellular disintegration features," Cell Death and Differentiation, vol. 17, no. 6, pp. 922-930, 2010.

[81] H. Kono and K. L. Rock, "How dying cells alert the immune system to danger," Nature Reviews Immunology, vol. 8, no. 4, pp. 279-289, 2008.

[82] S. He, L. Wang, L. Miao et al., "Receptor interacting protein kinase-3 determines cellular necrotic response to TNF- $\alpha$," Cell, vol. 137, no. 6, pp. 1100-1111, 2009.

[83] S. He, Y. Liang, F. Shao, and X. Wang, "Toll-like receptors activate programmed necrosis in macrophages through a receptor-interacting kinase-3-mediated pathway," Proceedings of the National Academy of Sciences of the United States of America, vol. 108, no. 50, pp. 20054-20059, 2011.

[84] D.-W. Zhang, J. Shao, J. Lin et al., "RIP3, an energy metabolism regulator that switches TNF-induced cell death from apoptosis to necrosis," Science, vol. 325, no. 5938, pp. 332-336, 2009.

[85] P. Vandenabeele, W. Declercq, F. Van Herreweghe, and T. Vanden Berghe, "The role of the kinases RIP1 and RIP3 in TNFinduced necrosis," Science Signaling, vol. 3, no. 115, pp. 3115-3124, 2010.

[86] C. Günther, B. Buchen, G. W. He et al., "Caspase- 8 controls the gut response to microbial challenges by Tnf- $\alpha$-dependent and independent pathways," Gut, vol. 64, no. 4, pp. 601-610, 2015.

[87] O. Micheau and J. Tschopp, "Induction of TNF receptor Imediated apoptosis via two sequential signaling complexes," Cell, vol. 114, no. 2, pp. 181-190, 2003.

[88] H. Wang, L. Sun, L. Su et al., "Mixed lineage kinase domainlike protein MLKL causes necrotic membrane disruption upon phosphorylation by RIP3," Molecular Cell, vol. 54, no. 1, pp. 133146, 2014.

[89] Z. Zhou, V. Han, and J. Han, "New components of the necroptotic pathway," Protein and Cell, vol. 3, no. 11, pp. 811-817, 2012.

[90] J. Li, T. McQuade, A. B. Siemer et al., “The RIP1/RIP3 necrosome forms a functional amyloid signaling complex required for programmed necrosis," Cell, vol. 150, no. 2, pp. 339-350, 2012.

[91] J. Zhao, S. Jitkaew, Z. Cai et al., "Mixed lineage kinase domainlike is a key receptor interacting protein 3 downstream component of TNF-induced necrosis," Proceedings of the National Academy of Sciences of the United States of America, vol. 109, no. 14, pp. 5322-5327, 2012.

[92] L. Sun, H. Wang, Z. Wang et al., "Mixed lineage kinase domainlike protein mediates necrosis signaling downstream of RIP3 kinase," Cell, vol. 148, no. 1-2, pp. 213-227, 2012.

[93] Y. Dondelinger, W. Declercq, S. Montessuit et al., "MLKL compromises plasma membrane integrity by binding to phosphatidylinositol phosphates," Cell Reports, vol. 7, no. 4, pp. 971981, 2014.

[94] J. M. Murphy, P. E. Czabotar, J. M. Hildebrand et al., “The pseudokinase MLKL mediates necroptosis via a molecular switch mechanism," Immunity, vol. 39, no. 3, pp. 443-453, 2013.

[95] Z. Cai, S. Jitkaew, J. Zhao et al., "Plasma membrane translocation of trimerized MLKL protein is required for TNF-induced necroptosis," Nature Cell Biology, vol. 16, no. 1, pp. 55-65, 2014.

[96] J. M. Hildebrand, M. C. Tanzer, I. S. Lucet et al., "Activation of the pseudokinase MLKL unleashes the four-helix bundle domain to induce membrane localization and necroptotic cell death," Proceedings of the National Academy of Sciences of the United States of America, vol. 111, no. 42, pp. 15072-15077, 2014.
[97] W. J. Kaiser, J. W. Upton, A. B. Long et al., "RIP3 mediates the embryonic lethality of caspase-8-deficient mice," Nature, vol. 471, no. 7338, pp. 368-372, 2011.

[98] A. Polykratis, N. Hermance, M. Zelic et al., "Cutting edge: RIPK1 kinase inactive mice are viable and protected from TNFInduced necroptosis in vivo," The Journal of Immunology, vol. 193, no. 4, pp. 1539-1543, 2014.

[99] A. Kaczmarek, P. Vandenabeele, and D. V. Krysko, "Necroptosis: the release of damage-associated molecular patterns and its physiological relevance," Immunity, vol. 38, no. 2, pp. 209-223, 2013.

[100] W. Zhou and J. Yuan, "Necroptosis in health and diseases," Seminars in Cell and Developmental Biology, vol. 35, pp. 14-23, 2014.

[101] C. Günther, B. Buchen, M. F. Neurath, and C. Becker, "Regulation and pathophysiological role of epithelial turnover in the gut," Seminars in Cell and Developmental Biology, vol. 35, pp. 40-50, 2014.

[102] M. Pasparakis and P. Vandenabeele, "Necroptosis and its role in inflammation," Nature, vol. 517, no. 7534, pp. 311-320, 2015.

[103] M. Pierdomenico, A. Negroni, L. Stronati et al., "Necroptosis is active in children with inflammatory bowel disease and contributes to heighten intestinal inflammation," The American Journal of Gastroenterology, vol. 109, no. 2, pp. 279-287, 2014.

[104] M. A. Brennan and B. T. Cookson, "Salmonella induces macrophage death by caspase-1-dependent necrosis," Molecular Microbiology, vol. 38, no. 1, pp. 31-40, 2000.

[105] K. E. Lawlor, N. Khan, A. Mildenhall et al., "RIPK3 promotes cell death and NLRP3 inflammasome activation in the absence of MLKL," Nature Communications, vol. 6, article 6282, 2015.

[106] V. A. K. Rathinam, S. K. Vanaja, and K. A. Fitzgerald, "Regulation of inflammasome signaling," Nature Immunology, vol. 13, no. 4, pp. 333-342, 2012.

[107] E. A. Miao, J. V. Rajan, and A. Aderem, "Caspase-1-induced pyroptotic cell death," Immunological Reviews, vol. 243, no. 1, pp. 206-214, 2011.

[108] T. Bergsbaken, S. L. Fink, and B. T. Cookson, "Pyroptosis: host cell death and inflammation," Nature Reviews Microbiology, vol. 7, no. 2, pp. 99-109, 2009.

[109] P. Broz and D. M. Monack, "Noncanonical inflammasomes: caspase-11 activation and effector mechanisms," PLoS Pathogens, vol. 9, no. 2, Article ID e1003144, 2013.

[110] M. Lamkanfi and V. M. Dixit, "Mechanisms and functions of inflammasomes," Cell, vol. 157, no. 5, pp. 1013-1022, 2014.

[111] T.-B. Kang, S.-H. Yang, B. Toth, A. Kovalenko, and D. Wallach, "Caspase-8 blocks kinase RIPK3-mediated activation of the NLRP3 inflammasome," Immunity, vol. 38, no. 1, pp. 27-40, 2013.

[112] F. S. Sutterwala, S. Haasken, and S. L. Cassel, "Mechanism of NLRP3 inflammasome activation," Annals of the New York Academy of Sciences, vol. 1319, no. 1, pp. 82-95, 2014.

[113] B. Ey, A. Eyking, M. Klepak et al., "Loss of TLR2 worsens spontaneous colitis in MDR1A deficiency through commensally induced pyroptosis," Journal of Immunology, vol. 190, no. 11, pp. 5676-5688, 2013.

[114] S. L. Fink and B. T. Cookson, "Pyroptosis and host cell death responses during Salmonella infection," Cellular Microbiology, vol. 9, no. 11, pp. 2562-2570, 2007.

[115] T. Fernandes-Alnemri, J. Wu, J.-W. Yu et al., "The pyroptosome: a supramolecular assembly of ASC dimers mediating inflammatory cell death via caspase-1 activation," Cell Death and Differentiation, vol. 14, no. 9, pp. 1590-1604, 2007. 
[116] E. Elinav, T. Strowig, A. L. Kau et al., "NLRP6 inflammasome regulates colonic microbial ecology and risk for colitis," Cell, vol. 145, no. 5, pp. 745-757, 2011.

[117] J. Dupaul-Chicoine, G. Yeretssian, K. Doiron et al., "Control of intestinal homeostasis, colitis, and colitis-associated colorectal cancer by the inflammatory caspases," Immunity, vol. 32, no. 3 , pp. 367-378, 2010.

[118] T. M. Ng and D. M. Monack, "Revisiting caspase-11 function in host defense," Cell Host and Microbe, vol. 14, no. 1, pp. 9-14, 2013.

[119] D. Demon, A. Kuchmiy, A. Fossoul, Q. Zhu, T.-D. Kanneganti, and M. Lamkanfi, "Caspase-11 is expressed in the colonic mucosa and protects against dextran sodium sulfate-induced colitis," Mucosal Immunology, vol. 7, no. 6, pp. 1480-1491, 2014.

[120] B. Levine and D. J. Klionsky, "Development by self-digestion: molecular mechanisms and biological functions of autophagy," Developmental Cell, vol. 6, no. 4, pp. 463-477, 2004.

[121] S. W. Ryter, K. Mizumura, and A. M. K. Choi, "The impact of autophagy on cell death modalities," International Journal of Cell Biology, vol. 2014, Article ID 502676, 12 pages, 2014.

[122] L. Galluzzi, I. Vitale, J. M. Abrams et al., "Molecular definitions of cell death subroutines: recommendations of the Nomenclature Committee on Cell Death 20122," Cell Death and Differentiation, vol. 19, no. 1, pp. 107-120, 2012.

[123] N. Mizushima, B. Levine, A. M. Cuervo, and D. J. Klionsky, "Autophagy fights disease through cellular self-digestion," Nature, vol. 451, no. 7182, pp. 1069-1075, 2008.

[124] T. Nunes, C. Bernardazzi, and H. S. de Souza, "Cell death and inflammatory bowel diseases: apoptosis, necrosis, and autophagy in the intestinal epithelium," BioMed Research International, vol. 2014, Article ID 218493, 12 pages, 2014.

[125] N. Mizushima and B. Levine, "Autophagy in mammalian development and differentiation," Nature Cell Biology, vol. 12, no. 9, pp. 823-830, 2010.

[126] W. E. Dowdle, B. Nyfeler, J. Nagel et al., "Selective VPS34 inhibitor blocks autophagy and uncovers a role for NCOA4 in ferritin degradation and iron homeostasis in vivo," Nature Cell Biology, vol. 16, no. 11, pp. 1069-1079, 2014.

[127] J. L. Benjamin, R. Sumpter Jr., B. Levine, and L. V. Hooper, "Intestinal epithelial autophagy is essential for host defense against invasive bacteria," Cell Host and Microbe, vol. 13, no. 6, pp. 723-734, 2013.

[128] K. Cadwell, J. Y. Liu, S. L. Brown et al., "A key role for autophagy and the autophagy gene Atg1611 in mouse and human intestinal Paneth cells," Nature, vol. 456, no. 7219, pp. 259-263, 2008.

[129] T. Saitoh, N. Fujita, M. H. Jang et al., "Loss of the autophagy protein Atg16L1 enhances endotoxin-induced IL-1 $\beta$ production," Nature, vol. 456, no. 7219, pp. 264-268, 2008.

[130] N. Dupont, S. Jiang, M. Pilli, W. Ornatowski, D. Bhattacharya, and V. Deretic, "Autophagy-based unconventional secretory pathway for extracellular delivery of IL-1 $\beta$," EMBO Journal, vol. 30, no. 23, pp. 4701-4711, 2011. 


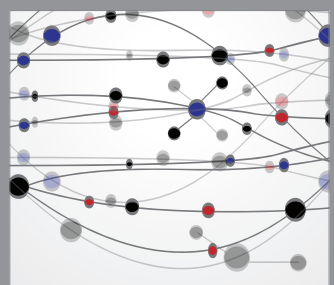

The Scientific World Journal
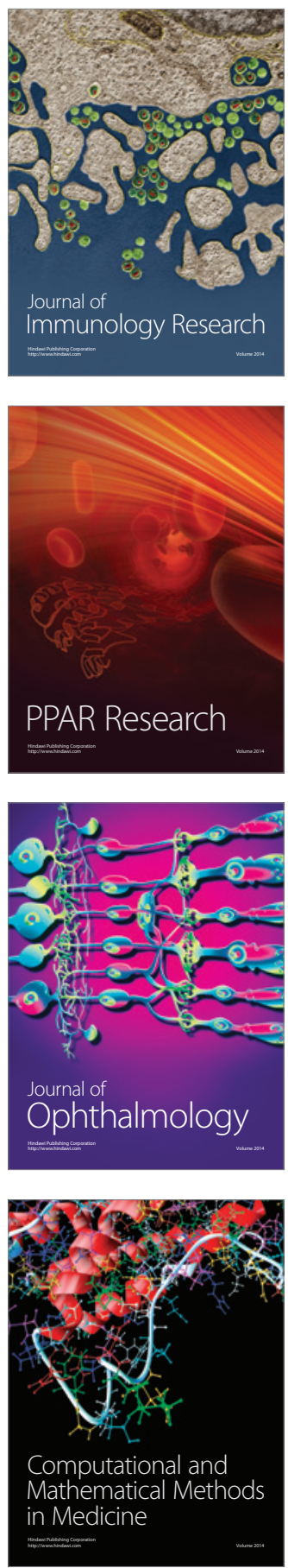

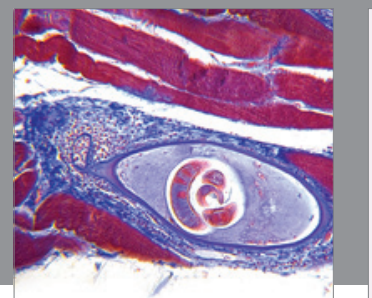

Gastroenterology

Research and Practice
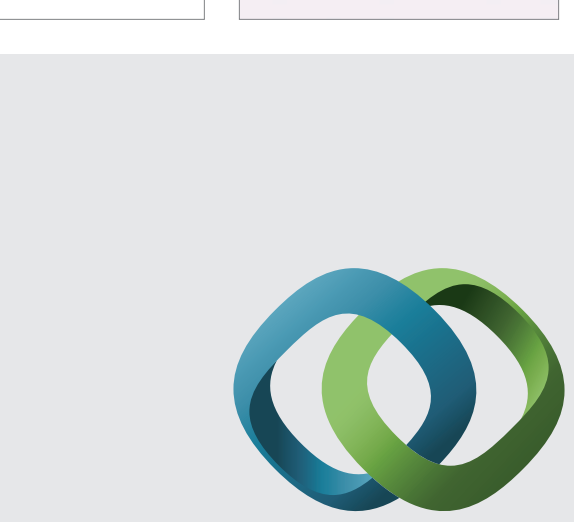

\section{Hindawi}

Submit your manuscripts at

http://www.hindawi.com
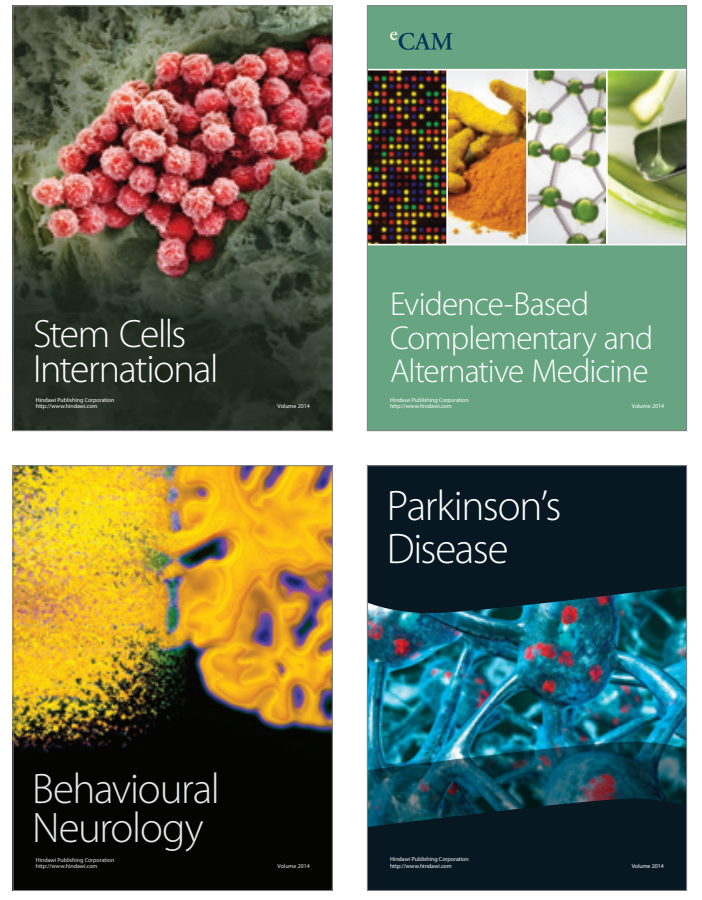
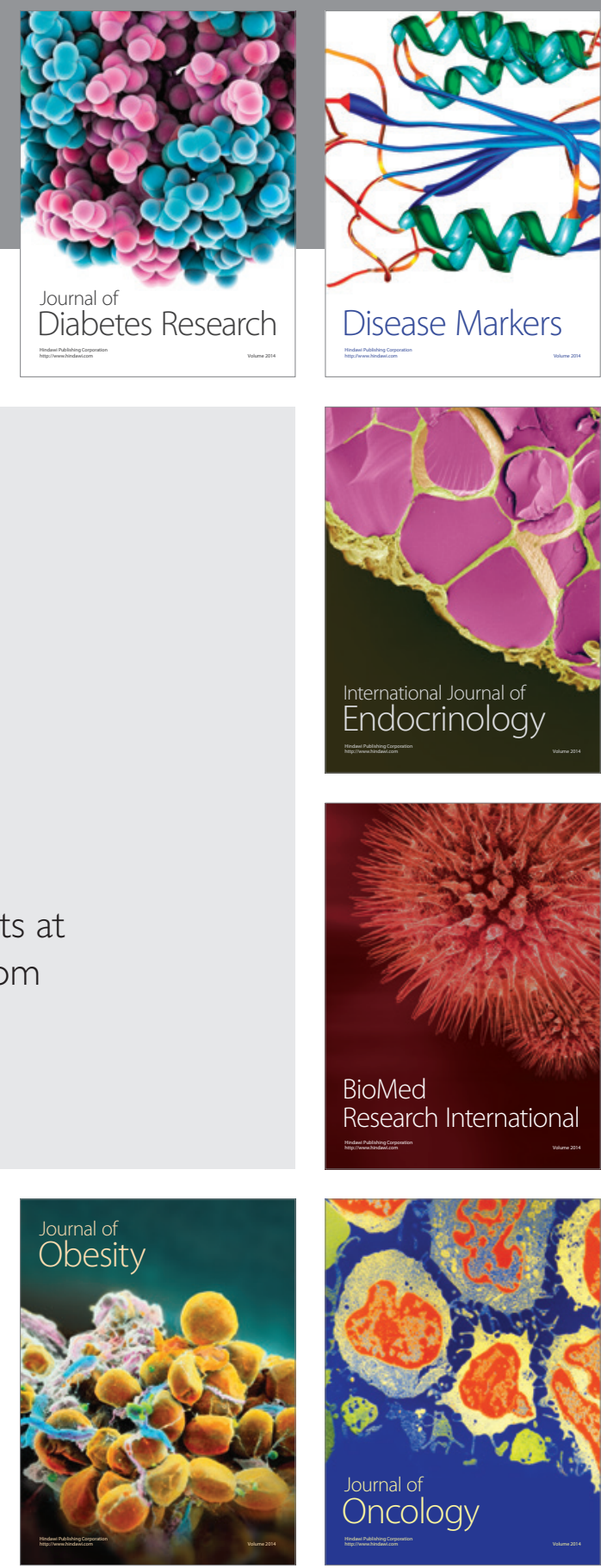

Disease Markers
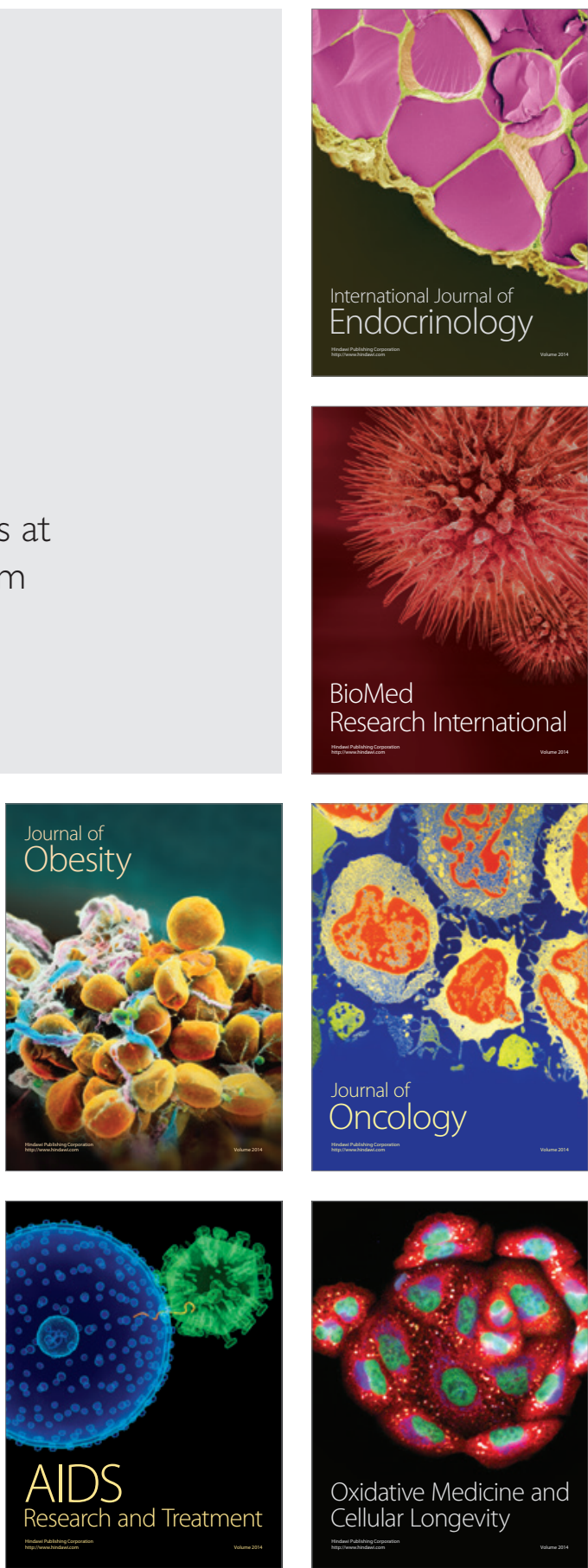